\title{
Plant-Made Vaccines
}

\section{Kathleen L Hefferon*}

Cell and Systems Biology, University of Toronto, 25 Willcocks Street, Toronto, Ontario, Canada

Plants offer enormous potential as production platforms for vaccines and therapeutic proteins. Plant-derived vaccines, for example, present an alternative to conventional vaccines by facilitating safe and effective oral delivery through consumption of edible plant tissue. Many infectious diseases enter the body through mucosal surfaces such as the gut, and as a result, vaccines expressed in the form of edible plant tissues offer a select advantage. The plant tissues can protect the antigen as it passes through the digestive tract. Plants are capable of producing recombinant antigens that undergo similar posttranslational modifications as their mammalian-derived counterparts and in contrast to bacterial expression systems. Moreover, high yields of biopharmaceuticals can be obtained, depending on the specific plant production platform. After these purification steps, the cost of producing plant-derived proteins represents only a fraction of the cost of proteins produced from analogous mammalian cell culture systems.

Proteins derived from plant expression systems differ from derived and mammalian cell cultures with respect to their respective glycosylation motifs. Many mammalian therapeutic proteins are glycoproteins and possess specific $\mathrm{N}$ - and O-glycosylation motifs not found in plants, potentially leading to adverse effects such as increases in allergenicity. It is now possible to glyco-engineer plants to more closely resemble their mammalian counterparts by altering glycosylation pathways which predominate in plant but not mammalian cells. Humanization of plant made vaccines and therapeutic proteins can be accomplished by incorporating the necessary glycosylation pathways in transgenic plants, as well as producing knockout plants which no longer produce the specific glycosylation motifs prone to cause adverse effects in humans. Therapeutic plant proteins are also constructed which can accumulate in the endoplasmic reticulum of plant cells; in this way, they can avoid undesired translational modifications as well as retain their stability in a plant cellular environment.

While transformation is one approach for the generation of biopharmaceutical proteins in plants, transient expression systems may also be used. Plant virus expression vector systems and agroinfection can produce extremely large quantities of therapeutic proteins within a short period of time (from a few days to a week or two, depending on the virus/host system). Plant viruses can be engineered to either express a full-length foreign protein or a vaccine epitope which is displayed on the viral surface by generating chimeras with the coat protein. Agroinfection involves dipping non-transformed plants into a solution of Agrobacteria harbouring the pharmaceutical gene of interest. Selection of which plant expression platform for pharmaceutical protein production to use is based upon determining the optimal plant species, whether it be whole plant or cell culture, and whether stable transformation or transient expression best fits the nature of the therapeutic protein under investigation and its proposed applications.

One of the major driving forces behind the use of plants as delivery systems for therapeutic protein production has been to rapidly produce cost-effective, safe and easily transportable vaccines to combat infectious diseases which are major causes of mortality in developing countries. The first proof of concept edible vaccine was generated against Hepatitis B virus, and was demonstrated to be efficacious at generating an immune response in human volunteers. Other examples have followed suit. Two of the most devastating diarrheal diseases for children residing in the Third World are enterotoxigenic E. coli (ETEC) and Norwalk virus or Norovirus (NV). Preliminary clinical trials involved feeding transgenic potato or corn expressing either LT-B or $\mathrm{NV}$ to healthy adult volunteers in a randomized, double-blind fashion. Initial studies involving direct consumption of antigens within edible plant tissues indicated that substantial IgG and IgA responses could be induced, and protection against challenge with the pathogens could be observed. Vaccines and monoclonal antibodies for other diseases known to be major challenges for developing countries, such as Human papillomavirus, and even Human immunodeficiency virus, are under development using plant production platforms. Plant-derived vaccines can also be used to combat so-called orphan diseases, such as rabies or hookworm, whose cures are inadequately funded in many parts of the world.

There are plenty of other motivations for producing biopharmaceuticals using plant expression platforms. In the United States, for example, efforts are being made to generate vaccines to combat global pandemics, such as Influenza virus. This year, the GreenVax Project of the US is utilizing a plant-based expression system to produce the H1N1 swine flu vaccine rapidly and in large quantities, to be stockpiled and on hand for potential future pandemics. Along the same lines, plant-derived vaccines against potential bioterrorism agents, such as anthrax, smallpox and the plague are under development.

A new application for plant-derived vaccines has been in the emerging field of personalized medicine. For example, NonHodgkins Disease (NHL), a cancer involving the over proliferation of B-lymphocytes, is a major killer in North America. Each NHL patient expresses his/her own individual idiotype on these over proliferating cells. The fact that plants can produce vaccine antigens cheaply and rapidly makes them ideal candidates for combating cancers such as these. Using a plant production platform, high levels of idiotype can be produced and reintroduced as a vaccine to NHL patients, thus providing them with an opportunity to stop the disease in its tracks shortly after diagnosis, a feat that was not feasible using conventional vaccination strategies.

Plant-derived vaccines continue to provide hope for more immunogenic, more effective and less expensive vaccination strategies against mucosal pathogens. The time has come for plant-derived biopharmaceuticals to make their mark.

*Corresponding author: Kathleen L Hefferon, Cell and Systems Biology, University of Toronto, 25 Willcocks Street, Toronto, Ontario, Canada, Tel: (607) 387 6304; Fax: (607) 254-5454; E-mail: Kathleen.hefferon@alumni.utoronto.ca

Received August 22, 2012; Accepted August 23, 2012; Published August 24 2012

Citation: Hefferon KL (2012) Plant-Made Vaccines. J Vaccines Vaccin 3:e108. doi:10.4172/2157-7560.1000e108

Copyright: (c) 2012 Hefferon KL. This is an open-access article distributed under the terms of the Creative Commons Attribution License, which permits unrestricted use, distribution, and reproduction in any medium, provided the original author and source are credited. 